\title{
Impacts of online and group perinatal education: a mixed methods study protocol for the optimization of perinatal health services
}

Geneviève Roch ${ }^{1,2,3^{*}}$, Roxane Borgès Da Silva ${ }^{4,5}$, Francine de Montigny ${ }^{6}$, Holly O. Witteman ${ }^{2,7}$, Tamarha Pierce ${ }^{8}$, Sonia Semenic ${ }^{9}$, Julie Poissant ${ }^{10}$, André-Anne Parent ${ }^{11}$, Deena White ${ }^{12}$, Nils Chaillett ${ }^{4,13}$, Carl-Ardy Dubois ${ }^{4,14}$, Mathieu Ouimet ${ }^{2,13}$, Geneviève Lapointe ${ }^{1,2,3}$, Stéphane Turcotte ${ }^{3}$, Alexandre Prud'homme ${ }^{4}$, Geneviève Painchaud Guérard ${ }^{2}$ and Marie-Pierre Gagnon ${ }^{1,2}$

\begin{abstract}
Background: Prenatal education is a core component of perinatal care and services provided by health institutions. Whereas group prenatal education is the most common educational model, some health institutions have opted to implement online prenatal education to address accessibility issues as well as the evolving needs of future parents. Various studies have shown that prenatal education can be effective in acquisition of knowledge on labour and delivery, reducing psychological distress and maximising father's involvement. However, these results may depend on educational material, organization, format and content. Furthermore, the effectiveness of online prenatal education compared to group prenatal education remains unclear in the literature. This project aims to evaluate the impacts of group prenatal education and online prenatal education on health determinants and users' health status, as well as on networks of perinatal educational services maintained with community-based partners.

Methods: This multipronged mixed methods study uses a collaborative research approach to integrate and mobilize knowledge throughout the process. It consists of: 1) a prospective cohort study with quantitative data collection and qualitative interviews with future and new parents; and 2) a multiple case study integrating documentary sources and interviews with stakeholders involved in the implementation of perinatal information service networks and collaborations with community partners. Perinatal health indicators and determinants will be compared between prenatal education groups (group prenatal education and online prenatal education) and standard care without these prenatal education services (control group).

(Continued on next page)
\end{abstract}

\footnotetext{
* Correspondence: genevieve.roch@fsi.ulaval.ca

${ }^{1}$ Faculty of Nursing, Université Laval, 1050 avenue de la Médecine, Québec,

QC GIV 0A6, Canada

${ }^{2} \mathrm{CHU}$ de Québec Research Centre - Université Laval, Hôpital Saint-François

d'Assise, 10 rue de l'Espinay, Québec, QC G1L 3L5, Canada

Full list of author information is available at the end of the article
}

(c) The Author(s). 2018 Open Access This article is distributed under the terms of the Creative Commons Attribution 4.0 International License (http://creativecommons.org/licenses/by/4.0/), which permits unrestricted use, distribution, and reproduction in any medium, provided you give appropriate credit to the original author(s) and the source, provide a link to the Creative Commons license, and indicate if changes were made. The Creative Commons Public Domain Dedication waiver (http://creativecommons.org/publicdomain/zero/1.0/) applies to the data made available in this article, unless otherwise stated. 
(Continued from previous page)

Discussion: This study will provide knowledge about the impact of online prenatal education as a new technological service delivery model compared to traditional group prenatal education. Indicators related to the complementarity of these interventions and those available in community settings will refine our understanding of regional perinatal services networks. Results will assist decision-making regarding service organization and delivery models of prenatal education services.

Protocol version: Version 1 (February 9 2018).

Keywords: Prenatal education, Perinatal care, Pregnancy, Childbirth education, Online education, Community health networks, Community health services, Health status indicators, Mixed methods

\section{Background}

Prenatal information is a decisive determinant of health choices made by pregnant women and their partners as they move through the continuum of perinatal services $[1$, 2]. Considering the myriad of information sources publicly available and their variable quality [3-7], prenatal education remains a health promotion strategy at the core of perinatal care and services provided by health and social services centers [8-10] [S-10] and is supported by public policies $[10,11]$. Group prenatal education is one of the most common educational models [12]. Various studies have shown that group prenatal education can be effective in the preparation for labour and delivery, reducing anxiety and maximising partners' involvement. However, these results depend on the organization, format, and content of the educational services [13-17]. In order to address accessibility issues as well as the evolving needs of future parents, some health and social services centers have opted to recommend or implement online prenatal education, while still offering group prenatal education. Decision makers, however, are concerned about the impacts of this new educational mode on the efficacy of health services networks. In a restructuring context where the deployment of online education opens the door to new complementary prenatal education to group education, it is important to understand the contribution of these two educational modes on health determinants and users' perinatal health [18]. Because of the heterogeneity of delivery modes [9], evidence of prenatal education effectiveness and impact is scarce or contradictory for group prenatal education $[12,14-17,19]$ and very limited for online prenatal education [20-22], although online education may address the needs of certain users and improve accessibility [23-29]. Within a health promotion context, prenatal education delivered by health and social services centers could be improved by being integrated into a continuum of perinatal information in partnership with existing community services networks [30]. Several studies show that networking may contribute to health system effectiveness, but structural characteristics and collaborations with community partners surrounding prenatal education and information remain unknown [31-33]. There is thus an urgent need to collect robust data on the impacts of group prenatal education and online prenatal education, and to consolidate perinatal information networks with community partners.

The aim of this project is to evaluate the impacts of group prenatal education and online prenatal education provided or recommended by health and social services centers on health determinants and users' health status, as well as on networks of perinatal educational services maintained with community-based partners. Specific objectives are to: 1) document the characteristics of group prenatal education and online prenatal education and contribute to their optimization; 2) evaluate the impacts of group prenatal education and online prenatal education on health determinants and the perinatal health status of parents; 3) evaluate characteristics and collaborations related to perinatal educational services within which group prenatal education and online prenatal education are offered, with community-based partners.

\section{Methods}

This multipronged study uses convergent mixed methods through a collaborative research approach to integrate and mobilize knowledge [34, 35]. More precisely, it will consist of 1) a prospective cohort study with quantitative data collection and qualitative interviews with future and new parents and 2) a multiple case study integrating documentary sources and interviews with stakeholders involved in the implementation of perinatal information service networks and collaborations with community partners. The complementarity of the quantitative and qualitative data will provide a broader perspective on perinatal information sources and networks in order to evaluate the impacts of prenatal education.

\section{Participating sites}

The study will be conducted within the geographic territories covered by two health and social services centers 
located in adjacent regions in the province of Québec, Canada, with an approximate total area of $34,000 \mathrm{~km}^{2}$ and total population of $1,162,000$ inhabitants. Created in 2015, these regional institutions are responsible for the provision of health care and services within their territories and for binding agreements with partner organizations (e.g. community organizations, medical clinics, network clinics, etc.) [36]. The health and social services centers are providing similar group prenatal education, with some variations related to their resources and specific population needs. Both are currently using an online prenatal education interface developed by a private provider [37]. These institutions also have access to a perinatal information source developed by the Ministry of Health and Social Services of Québec to maintain the harmonization of content [38].

\section{Participants}

For the cohort study, women will be eligible if they: a) are at the beginning of their pregnancy (10 to 20 weeks); b) live within the targeted geographic territories; c) are fluent in French; d) have not given birth previously and e) have a valid email address and access to internet. Male and female partners of women meeting these criteria will be eligible as partners. Partners who already had children with another woman will also be eligible. For qualitative interviews, parents will be eligible if they: a) have a 6- to 12-week-old infant; b) attended group prenatal education or online prenatal education recommended by participant sites; c) live within the targeted geographic territories; d) are fluent in French. For the multiple case study, prenatal education stakeholders (managers and health professionals) will be eligible if they: a) are working within the participating sites or related services networks; b) are interested in sharing their understanding of structural characteristics and determinants of collaboration between health and social services centers and community partners involved in group prenatal education and online prenatal education offer; and c) have been in their position for at least 3 months. These stakeholders will be identified with the help of collaborators from participating sites.

\section{Outcomes}

Primary and secondary outcomes related to perinatal health and perinatal health determinants were identified from a literature review on group prenatal education effects $[18,39]$. Based on studies that demonstrated significant effects of group prenatal education, the main outcome for health determinant is perinatal knowledge $[13,40]$ and will be measured with an adapted version of the Health Pregnancies Knowledge Survey [41]. The questionnaire will be adapted in collaboration with prenatal education trainers from the different participant training sites, in order to ensure that all knowledge items are covered in group and online prenatal education.

The main secondary outcome is psychological distress, measured with a validated French version of the 12-item General Health Questionnaire [42] and considered as the most important outcome for perinatal health measures (i.e., main outcome measuring a health determinant). Other secondary outcomes include: breastfeeding self-efficacy, assessed with a French version of the Breastfeeding Self-Efficacy Scale Short-Form [43, 44]; anxiety, assessed with a validated French version of the State-Trait Anxiety Inventory [45]; self-efficacy in the parenting role, assessed with a French version of the Parent Expectations Survey [46, 47]; depression, assessed with a validated French version of the Edinburgh Postnatal Depression Scale [48, 49]; concern about labour and birth, assessed with a French version of the Oxford Worries about Labour Scale [50]; control during childbirth, assessed with a French version of the Labour Agentry Scale [51]; personal control in pain relief during childbirth, assessed with a French version of the Personal Control in Pain Relief Scale [52], breastfeeding status and birth weight. A back translation process [53, 54] will be used to translate English versions of the Oxford Worries about Labour Scale, Labour Agentry Scale and Personal Control in Pain Relief Scale to French. Data on sociodemographic characteristics, pregnancy and childbirth history, and prenatal information sources consulted during pregnancy will also be collected as potential confounding factors. All questionnaires will be pre-tested with a test-retest procedure in order to assess their reliability [55].

\section{Data collection \\ Administrative data collection}

Throughout the entire duration of the project, administrative data needed to establish a general portrait of prenatal education use will be collected and updated with health and social services centers managers. These data will include different characteristics of the organization, format and content such as number and duration of group prenatal education meetings, health professionals involved in group prenatal education, themes covered in group prenatal education and online prenatal education, mode and fees for accessing group prenatal education and online prenatal education, and sources used for the development of group prenatal education and online prenatal education. Administrative data will also be obtained from the online prenatal education provider and will include access data, registration data and users' satisfaction data. 
Table 1 Distribution of outcomes measures through time

\begin{tabular}{|c|c|c|c|c|c|c|}
\hline & \multicolumn{2}{|c|}{ (T1) 10-20 weeks of pregnancy } & \multicolumn{2}{|c|}{ (T2) 33 weeks of pregnancy } & \multicolumn{2}{|c|}{ (T3) 6 weeks after child birth } \\
\hline & Pregnant women & Partners & Pregnant women & Partners & Mothers & Partners \\
\hline \multicolumn{7}{|l|}{ Main outcome measure } \\
\hline Health Pregnancies Knowledge Survey & $\checkmark$ & $\checkmark$ & $\checkmark$ & $\checkmark$ & & \\
\hline \multicolumn{7}{|l|}{ Secondary outcomes measures } \\
\hline General Health Questionnaire & $\checkmark$ & $\checkmark$ & $\checkmark$ & $\checkmark$ & $\checkmark$ & $\checkmark$ \\
\hline Breastfeeding Self-Efficacy Scale & $\checkmark$ & $\checkmark$ & $\checkmark$ & $\checkmark$ & $\checkmark$ & $\checkmark$ \\
\hline State-Trait Anxiety Inventory & $\checkmark$ & $\checkmark$ & $\checkmark$ & $\checkmark$ & $\checkmark$ & $\checkmark$ \\
\hline Parent Expectations Survey & $\checkmark$ & $\checkmark$ & $\checkmark$ & $\checkmark$ & $\checkmark$ & $\checkmark$ \\
\hline Edinburgh Postnatal Depression Scale & $\checkmark$ & $\checkmark$ & $\checkmark$ & $\checkmark$ & $\checkmark$ & $\checkmark$ \\
\hline Sociodemographics characteristics & $\checkmark$ & $\checkmark$ & & & & \\
\hline Pregnancy history & & & $\checkmark$ & & & \\
\hline Prenatal information sources & & & $\checkmark$ & $\checkmark$ & & \\
\hline Childbirth history & & & & & $\checkmark$ & \\
\hline Breastfeeding status & & & & & $\checkmark$ & \\
\hline Oxford Worries about Labour Scale & & & & & $\checkmark$ & $\checkmark$ \\
\hline Labour Agentry Scale & & & & & $\checkmark$ & $\checkmark$ \\
\hline Birth weight & & & & & $\checkmark$ & $\checkmark$ \\
\hline
\end{tabular}

$\checkmark$ Outcome measured

\section{Time measurements (cohort study)}

Table 1 presents the distribution of outcomes measurements through time for the cohort study. Time measurements are calculated according to the continuum of services of participating institutions. The first questionnaire (T1) will be completed between the 10th and 20th week of pregnancy, in order to reach participants before the prenatal education period. The second questionnaire (T2) will be sent at 33 weeks of pregnancy, in order to reach participants after the prenatal education period. The third and last questionnaire (T3) will be sent 6 weeks after the expected date of birth. All questionnaires will be sent by email and completed online.

\section{Qualitative interviews}

Semi-structured individual qualitative interviews with parents will be based on an interview guide developed according to the Interactive Quality Health Education Outcomes Model [56]. Interviews will be conducted by phone in order to facilitate participation. Each interview will last approximately $45 \mathrm{~min}$. Mothers and partners from each participating site and each prenatal education mode (group or online prenatal education) will be recruited according to a stratified sampling until data saturation is reached (expected $N=40$ ) [57-59]. Qualitative interviews will be held simultaneously with the cohort study.

\section{Network data collection}

In order to evaluate structural characteristics of efficient networks and collaborations, individual qualitative interviews with prenatal education stakeholders will be held in the two participating health and social services centers and their related community-based organizations (expected $N=45$ ). The interview guide will be developed from a reference framework inspired by the work of Turrini et al. [31] for efficient network characteristics, and Lasker et al. [60] for partnerships functioning (Fig. 1). An adaptation of the Social Network Analysis Tool [61] will also be used in order to estimate how these characteristics and determinants may consolidate group prenatal education, online prenatal education and perinatal information. Each interview will last approximately $45 \mathrm{~min}$. These interviews will be held simultaneously with the cohort study and will be completed by documentary sources provided by the participating sites.

\section{Recruitment strategies}

For the cohort study, all questionnaires will be completed in electronic format and data kept on a secured server hosted by the principal investigator's institution. Pregnant women and their partners will be recruited at their first contact point with participating sites, namely at their first ultrasound test or prenatal meeting. In ultrasound clinics, a bookmark providing connection information will be given by receptionists when parents attend the first dating ultrasound. A research assistant will then be responsible to meet potential participants in the waiting room, provide them with the necessary information and give them the opportunity to complete the consent form and start answering the questionnaire on 


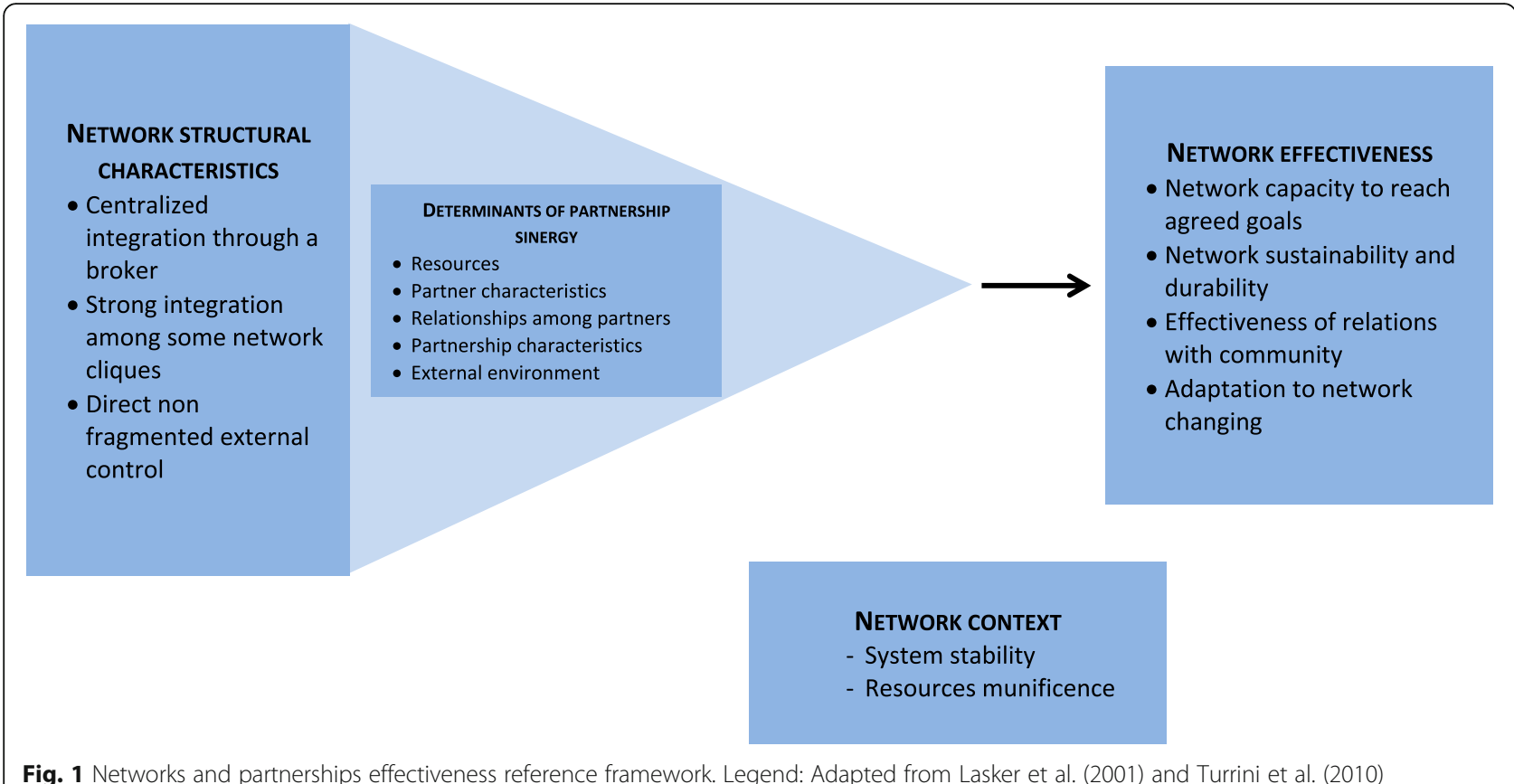

an iPad. Potential participants will also be free to keep the bookmark and complete the questionnaire later at home. In prenatal clinics, the bookmark and project information will be given by nurses to future parents, which will then be free to complete the online consent form and questionnaire at home. Posters will also be displayed in all participating sites, with the possibility for potential participants to contact the principal investigator or project coordinator directly if they want to participate. Once participants are registered, follow-up will be done by email or phone.

For qualitative interviews, parents will be recruited at their first postnatal clinic encounter in participating health and social services centers (e.g. immunization clinics, breastfeeding clinics, etc.). A bookmark with the research team coordinates and project information will be given by nurses to new parents, who will be invited to contact the research team in order to verify their eligibility and participate to the project.

For the multiple case study, expert stakeholders will be recruited through a snowball sampling technique starting with the health and social services centers' decision-makers initially involved in the study.

\section{Incentives and retention strategies}

In order to prevent loss to follow-up during the cohort study, automatic email reminders will be sent twice after the sending of $\mathrm{T} 1, \mathrm{~T} 2$ and $\mathrm{T} 3$ questionnaires. If the questionnaire is not completed after that, the research coordinator or a research assistant will call the participant as a last reminder or to record the reason for abandonment. All participants will be eligible for the drawing of six iPads, with chances to win proportionately increasing with the number of completed questionnaires (one to three).

\section{Statistical analysis Sample size}

Assuming an effect size of 0.36 (for perinatal knowledge with or without prenatal education) and a 1:1:2 allocation between groups (group prenatal education: online prenatal education: without prenatal education) [13], a power of $80 \%$ and a bilateral test threshold of 0.025 , a total of 445 pregnant women and 445 partners (2 groups of 111 with prenatal education and 1 group of 223 without prenatal education) is required at the third measurement time. An ongoing longitudinal study conducted by our research team with new parents in the Québec region allows us to expect a participation rate of $80 \%$ for partners and a retention rate of $70 \%$ at the end of the three measuring times. An initial sample size of 795 pregnant women and up to 795 partners is therefore anticipated. A second power calculation based on Jakubiec et al. data [40] was done for the most important secondary outcome (psychological distress) and resulted in a smaller sample size. Births by territory data suggest a sufficient pool to recruit the required sample size within 3 to 4 months.

\section{Quantitative analysis}

Descriptive analysis will be conducted at the three time points. For the main outcome (measured twice), 
difference between prenatal education groups (group prenatal education and online prenatal education) and standard care without these prenatal education services will be calculated. Bivariate linear regression models will then be used to measure the association between this difference and secondary outcomes. Bivariate linear regression models will be used to compare prenatal education groups (group prenatal education and online prenatal education) to the absence of these prenatal education services for health determinants measured twice. Non-multicollinearity, normality of residuals and homogeneity of variances will be verified and a variable transformation will be performed if these postulates are not met. Outcomes measured at the three time points will be analyzed with bivariate repeated measures models. For continuous and categorical outcomes, mixed models and generalized estimating equation models will be used respectively. For the generalized estimating equation models, binomial distribution will be used for binary outcomes and multinomial distribution for outcomes with multiple categories. Multiple imputation will be used for randomly distributed missing data. Depending on the results, sensitivity analysis may be performed for geographic regions, health establishments providing prenatal education, group or online prenatal education format, exposure level to online prenatal education and healthcare providers involved in pregnancy follow-up. All statistical models will be adjusted for sociodemographic data, use of other information sources and pregnancy follow-up data. Analysis will be performed with Statistical Analysis Software version 9.4 (SAS Institute, Cary, NC, USA).

\section{Qualitative analysis}

Data from semi-structured interviews will be recorded, transcribed, anonymized and analyzed with QDA Miner software version 5 (Provalis Research, Montreal, QC, Canada). Content analysis and integration of quantitative inferences will be conducted based on an adaptation of the Interactive Quality Health Education Outcomes Model [56]. Administrative data will be treated in a descriptive manner in order to establish the general and comparative profile of users. Quality and confidentiality of data will be rigorously ensured by the use of consolidated criteria and validated qualitative methods [62, 63].

\section{Multiple case study analysis}

Case studies will consist of perinatal information networks of the two participating health and social services centers in which local community perinatal information networks will be embedded. For each study case, matrices allowing the evaluation of determinants in relation to networks success factors and collaborative actions presented in perinatal governmental programs [64-66] will be developed alongside a content analysis [67]. UCINET software version 6 [68] will be used to view and compare perinatal networks structure according to the analytical approach described by Scott et al. [69], as recommended by Provan et al. for the reinforcement of efficient collaboration networks [61]. The integration of different data sources will allow a cross-sectional validation of results.

\section{Collaboration with decision-makers}

Decision-makers of the two participating health and social services centers have committed to facilitate the implementation of this project in their respective establishments. Based on the administrative data collected and usability of online prenatal education, they will standardize as much as possible their offer of group prenatal education and online prenatal education before the recruitment in order to optimize the results of the study. Responding to priorities in public health and clinical services organization, this engagement will facilitate a relevant follow-up of the impacts of group and online prenatal education. The study of the service delivery models for prenatal education and the associated regional networks providing these services will also promote collaboration between the political decision-makers of the Ministry of Health and Social Services of Québec, the National Public Health Institute of Québec, and the Public Health Agency of Canada who have agreed to actively participate in the interpretation of results and mobilization of knowledge strategies.

\section{Discussion}

\section{Knowledge translation strategies}

An advisory committee (composed of all authors, health professionals and managers as expert knowledge users) will support the development and operationalization of the study, notably for data collection follow-up and knowledge translation. A monitoring committee (composed of all authors, decision-makers, parent representatives and policy makers as expert knowledge users) will be responsible for sustained knowledge mobilization throughout all the study in order to support organizational and political decisions related to perinatal education services. This knowledge mobilization approach in its process, reflections, tools and results can be shared with the involved actors in order to disseminate the best practices in organizational terms for the users, the organizations, and partners of perinatal services networks. The use of brief reports, narrated slides and a website intended for the users, decision-makers, and partners will make up the principal knowledge transfer strategies and results dissemination. 


\section{Expected outcomes}

This study will be one of the first to consider the impacts of online prenatal education on different health determinants and perinatal health status in a Canadian context. This will allow for important knowledge acquisition regarding the impact of online prenatal education as a new technological service delivery model compared to an absence of group prenatal education in some health and social services centers settings. Indicators related to the complementarity of group and online prenatal education and those available in a community setting will refine our understanding of regional perinatal services networks. As studies involving future fathers or partners are uncommon, although their involvement in perinatal period is strongly recommended [70], results will also indicate how group and online prenatal education can contribute to their well-being and that of their family. This project also has the potential to improve harmonization of group prenatal education and the user-friendliness of online prenatal education. This could potentially improve nurses' professional practices, as well as those of other health professionals and community stakeholders involved in perinatal education. The partnership approach will assist in the development of a measurement culture and support decision-making regarding service organization and delivery models of prenatal education in Québec as well as other Canadian provinces where online prenatal education are provided, in order to optimize perinatal health services.

\section{Acknowledgements}

We thank the Public Health Agency of Canada, the Ministry of Health and Social Services of Québec, the participating health and social services centers and the online prenatal education private provider for their in-kind contribution to the project. We also thank all knowledge users involved in the study for their input on its relevance, their commitment to its facilitation and their support for integrated knowledge transfer strategies.

\section{Funding}

This study is funded by the Canadian Institutes of Health Research (CIHR) and the Fonds de recherche Santé - Québec (FRSQ). Funding Reference Number: PHE-148163. FD is Tier 2 Canada Research Chair in Family Psychosocial Health and is supported by a FRQS Research Scholar Senior Career Award. HW is supported by a FRQS Research Scholar Junior 2 Career Award. MPG is Tier 2 Canada Research Chair in Technology and Practice in Health.

\section{Authors' contributions}

GR, RBS, MPG, and FD contributed to the conception and design of the study and wrote the draft manuscript with GPG. HW, TP, SS, JP, AP, ST and GPG contributed to the design of the cohort study. AAP, DW, CAD and GL contributed to the design of the case study; $\mathrm{MO}$ provided expertise in knowledge transfer and network analysis. All authors reviewed, read and approved the final version of the manuscript.

\section{Ethics approval and consent to participate}

Ethics committee review approval has been obtained from the Multicenter Ethics Committee of the Centre intégré de santé et de services sociaux de Chaudière-Appalaches; approval number: MP-23-2017-405), with all protocol modifications being mandatory to report. All participants will provide electronic consent following a procedure approved by the ethics board.

\section{Competing interests}

The authors declare that they have no competing interests.

\section{Publisher's Note}

Springer Nature remains neutral with regard to jurisdictional claims in published maps and institutional affiliations.

\section{Author details}

${ }^{1}$ Faculty of Nursing, Université Laval, 1050 avenue de la Médecine, Québec, QC G1V 0A6, Canada. ${ }^{2} \mathrm{CHU}$ de Québec Research Centre - Université Laval, Hôpital Saint-François d'Assise, 10 rue de l'Espinay, Québec, QC G1L 3L5,

Canada. ${ }^{3}$ Centre intégré de santé et de services sociaux de

Chaudière-Appalaches, Hôtel-Dieu de Lévis, 143 rue Wolfe, Lévis, QC G6V

3Z1, Canada. ${ }^{4}$ Université de Montréal Public Health Research Institute, 7101

avenue du Parc, Montréal, QC H3N 1X9, Canada. ${ }^{5}$ Faculty of Nursing,

Université de Montréal, 2375, chemin de la Côte-Ste-Catherine, Montréal, QC H3T 1A8, Canada. ${ }^{6}$ Department of Nursing, Université du Québec en Outaouais, 283 boulevard Alexandre-Taché CP 1250, Gatineau, QC J8X 3X7, Canada. ${ }^{7}$ Faculty of Medicine, Université Laval, 1050 avenue de la Médecine, Québec City, QC GIV 0A6, Canada. ${ }^{8}$ School of Psychology, Université Laval, 2325 Allée des Bibliothèques, Québec City, QC G1V 0A6, Canada. ${ }^{9}$ Ingram School of Nursing, McGill University, 680 Sherbrooke West, Montréal, QC H3A 2M7, Canada. ${ }^{10}$ Institut national de santé publique du Québec, 945 av Wolfe, Québec City, QC G1V 5B3, Canada. "'School of Social Work, Université de Montréal, 3150 rue Jean-Brillant, Montréal, QC H3T 1N8, Canada.

${ }^{12}$ Département de sociologie, Université de Montréal, 3150 rue Jean-Brillant, Montréal, QC H3T 1N8, Canada. ${ }^{13}$ Department of Political Science, Faculty of Social Sciences, Université Laval, 1030 avenue des Sciences Humaines, Québec, QC G1V 0A6, Canada. ${ }^{14}$ School of Public Health, Université de Montréal, 7101 avenue du Parc, Montréal, QC H3N 1X9, Canada.

Received: 12 February 2018 Accepted: 14 May 2018

Published online: 29 May 2018

\section{References}

1. Martin CJH, Robb Y. Women's views about the importance of education in preparation for childbirth. Nurse Educ Pract. 2013;13(6):512-8.

2. Koehn ML. Childbirth education outcomes: an integrative review of the literature. J Perinat Educ. 2002;11(3):10.

3. Edmonds JK, Cwiertniewicz T, Stoll K. Childbirth education prior to pregnancy? Survey findings of childbirth preferences and attitudes among young women. J Perinat Educ. 2015;24(2):93-101.

4. McArdle A, et al. How pregnant women learn about foetal movements: sources and preferences for information. Women Birth. 2015;28(1):54-9.

5. Criss $\mathrm{S}$, et al. The role of health information sources in decision-making among Hispanic mothers during their Children's first 1000 days of life. Matern Child Health J. 2015:19(11):2536-43.

6. Grimes HA, Forster DA, Newton MS. Sources of information used by women during pregnancy to meet their information needs. Midwifery. 2014;30(1):e26-33.

7. Buultjens M, Robinson P, Milgrom J. Online resources for new mothers: opportunities and challenges for perinatal health professionals. J Perinat Educ. 2012;21(2):99-111.

8. Dumas L. Focus groups to reveal parents' needs for prenatal education. J Perinat Educ. 2002;11(3):1-9.

9. Direction régionale de santé publique. L'information en période prénatale: Pratiques et besoins des mères, des pères et des intervenantes en santé et services sociaux de la Capitale-Nationale. Québec: Agence de la santé et des services sociaux de la Capitale-Nationale; 2014. p. 150.

10. Ministère de la Santé et des Services sociaux. Politique de périnatalité 2008 2018. Québec: Direction des communications du ministère de la Santé et des Services sociaux du Québec; 2008. p. 174.

11. Public Health Agency of Canada. The Canada prenatal nutrition program : a decade of promoting the health of mothers, babies and communities. Ottawa: Her Majesty the Queen in Right of Canada, represented by the Minister of Health; 2007.

12. Tu A, Poissant J. Avis Sur les effets des rencontres prénatales de groupe. Montréal: Institut national de santé publique du Québec; 2015. p. 135.

13. Ickovics JR, et al. Group prenatal care and perinatal outcomes: a randomized controlled trial. Obstet Gynecol. 2007;110(2 Pt 1):330-9. 
14. Brixval CS, et al. The effect of antenatal education in small classes on obstetric and psycho-social outcomes - a systematic review. Syst Rev. 2015;4:20.

15. Ferguson S, Davis D, Browne J. Does antenatal education affect labour and birth? A structured review of the literature. Women Birth. 2013;26(1):e5-8.

16. Gagnon AJ, Sandall J. Individual or group antenatal education for childbirth or parenthood, or both Cochrane Database of Systematic Reviews; 2007. p. (3 Art. No.: CD002869).

17. Martínez-Galiano JM, Delgado-Rodríguez M. Effectiveness of the professional who carries out the health education program: perinatal outcomes. Int J Womens Health. 2014;6:329-34.

18. Ministère de la Santé et des Services sociaux. La santé et ses déterminants, mieux comprendre pour mieux agir. Québec: La Direction des communications du ministère de la Santé et des Services sociaux; 2012.

19. Centre de ressources Meilleur départ. La prestation de l'éducation prénatale en Ontario : un résumé des conclusions de l'étude. Toronto (Ontario): Centre de ressources Meilleur départ; 2015.

20. Salonen $\mathrm{AH}$, et al. Effectiveness of an internet-based intervention enhancing Finnish parents' parenting satisfaction and parenting self-efficacy during the postpartum period. Midwifery. 2011;27(6):832-41.

21. Salonen $\mathrm{AH}$, et al. Impact of an internet-based intervention on Finnish mothers' perceptions of parenting satisfaction, infant centrality and depressive symptoms during the postpartum year. Midwifery. 2014;30(1):112-22.

22. Huang $M Z$, et al. Evaluating effects of a prenatal web-based breastfeeding education programme in Taiwan. J Clin Nurs. 2007;16(8):1571-9.

23. Romano AM. A changing landscape: implications of pregnant women's internet use for childbirth educators. J Perinat Educ. 2007;16(4):18-24.

24. Mackert $\mathrm{M}_{\text {, et }}$ al. Including men in prenatal health: the potential of e-health to improve birth outcomes. Telemed J E Health. 2015;21(3):207-12.

25. Lagan BM, Sinclair M, George Kernohan W. Internet use in pregnancy informs Women's decision making: a web-based survey. Birth. 2010;37(2):106-15.

26. Huberty J, et al. Describing the use of the internet for health, physical activity, and nutrition information in pregnant women. Matern Child Health J. 2013;17(8):1363-72.

27. Larsson M. A descriptive study of the use of the internet by women seeking pregnancy-related information. Midwifery. 2009:25(1):14-20.

28. Niela-Vilen $\mathrm{H}$, et al. Internet-based peer support for parents: a systematic integrative review. Int J Nurs Stud. 2014;51(11):1524-37.

29. StGeorge JM, Fletcher RJ. Fathers online: learning about fatherhood through the internet. J Perinat Educ. 2011;20(3):154-62.

30. Jones J, Barry MM. Exploring the relationship between synergy and partnership functioning factors in health promotion partnerships. Health Promot Int. 2011;26(4):408-20.

31. Turrini A, et al. Networking literature about determinants of network effectiveness. Public Adm. 2010;88(2):528-50.

32. Provan KG, Milward HB. Do networks really work? A framework for evaluating public-sector organizational networks. Public Adm Rev. 2001; 61(4):414-23.

33. Provan KG, Milward HB. Health services delivery networks: what do we know and where should we be headed? Healthc Pap. 2006;7(2):32-6. discussion 68-75

34. Baumbusch $J$, et al. Pursuing common agendas: a collaborative model for knowledge translation between research and practice in clinical settings. Res Nurs Health. 2008;31(2):130-40.

35. Witteman $\mathrm{HO}$, et al. User-centered design and the development of patient decision aids: protocol for a systematic review. Syst Rev. 2015;4:11.

36. Santé et services sociaux - Québec. Network reorganization.; Available from: http://www.msss.gouv.qc.ca/en/reseau/reorganisation/portrait. Accessed 18 Dec 2017.

37. Solviweb, Une nouvelle vie - un programme prénatal en ligne pour la future maman, son/sa partenaire et sa famille. 2015.

38. Institut nationale de santé publique. Portail d'information périnatale. 2018 Available from: https://www.inspq.qc.ca/information-perinatale.

39. Public Health Agency of Canada. Perinatal health indicators for Canada 2013: a report of the Canadian perinatal surveillance system. Ottawa; 2013. p. 78. http:// publications.gc.ca/collections/collection_2014/aspc-phac/HP7-1-2013-eng.pdf.

40. Jakubiec $D$, et al. Effect of attending childbirth education classes on psychological distress in pregnant women measured by means of the general health questionnaire. Adv Clin Exp Med. 2014;23(6):953-7.

41. Godin $\mathrm{K}$, et al. Assessing public health prenatal education knowledge: findings from the LDPC healthy pregnancies project. Ontario: Woodstock; 2014.
42. Salama-Younes $M$, et al. Factor structure and internal consistency of the 12item general health questionnaire (GHQ-12) and the subjective vitality scale (VS), and the relationship between them: a study from France. Health Qual Life Outcomes. 2009:7:22.

43. Dennis CL. The breastfeeding self-efficacy scale: psychometric assessment of the short form. J Obstet Gynecol Neonatal Nurs. 2003;32(6):734-44.

44. Degrange $M$, et al. Is self-confidence a factor for successful breastfeeding? Arch Pediatr. 2015;22(7):708-17.

45. Gauthier J, Bouchard S. Adaptation canadienne-française de la forme révisée du State-Trait Anxiety Inventory de Spielberger. Can J Behav Sci. 1993;25(4):559-78.

46. Reece SM. The parent expectations survey: a measure of perceived selfefficacy. Clin Nurs Res. 1992;1(4):336-46.

47. Savard J. Efficacité parentale perçue par des mères primipares exposées ou non aux soins d'infirmières ayant reçu la formation spécifique au programme d'aide périnatale aux parents (PAPP), in Faculty of nursing. Quebec City: Université Laval; 1997. p. 142.

48. Cox JL, Holden JM, Sagovsky R. Detection of postnatal depression. Development of the 10-item Edinburgh postnatal depression scale. $\mathrm{Br} J$ Psychiatry. 1987;150:782-6.

49. Guedeney N, Fermanian J. Validation study of the French version of the Edinburgh postnatal depression scale (EPDS): new results about use and psychometric properties. Eur Psychiatry. 1998:13(2):83-9.

50. Redshaw M, et al. The Oxford worries about labour scale: women's experience and measurement characteristics of a measure of maternal concern about labour and birth. Psychol Health Med. 2009;14(3):354-66.

51. Hodnett ED, Simmons-Tropea DA. The labour Agentry scale: psychometric properties of an instrument measuring control during childbirth. Res Nurs Health. 1987;10(5):301-10.

52. McCrea BH, Wright ME. Satisfaction in childbirth and perceptions of personal control in pain relief during labour. J Adv Nurs. 1999;29(4):877-84.

53. Beaton $\mathrm{DE}$, et al. Guidelines for the process of cross-cultural adaptation of self-report measures. Spine (Phila Pa 1976). 2000;25(24):3186-91.

54. Massoubre $C$, et al. The translation of questionnaires and of tests: techniques and problems. Can J Psychiatr. 2002;47(1):61-7.

55. Marx RG, et al. A comparison of two time intervals for test-retest reliability of health status instruments. J Clin Epidemiol. 2003;56(8):730-5.

56. Humenick SS. Program evaluation. In: Childbirth education: practice, research and theory. Philadelphia: WB Saunders Company; 2000. p. 593-608.

57. Sandelowski M. Focus on research methods combining qualitative and quantitative sampling, data collection, and analysis techniques. Res Nurs Health. 2000;23:246-55.

58. O'Reilly M, Parker N. Unsatisfactory saturation: a critical exploration of the notion of saturated sample sizes in qualitative research. Qual Res. 2012;13(2):190-7.

59. Hsieh H-F, Shannon SE. Three approaches to qualitative content analysis. Qual Health Res. 2005;15(9):1277-88.

60. Lasker RD, Weiss ES, Miller R. Partnership synergy: a practical framework for studying and strengthening the collaborative advantage. Milbank Q. 2001; 79(2):179

61. Provan $\mathrm{KG}$, et al. The use of network analysis to strengthen community partnerships. Public Adm Rev. 2005:65(5):603-13.

62. O'Cathain A, Murphy E, Nicholl J. The quality of mixed methods studies in health services research. J Health Serv Res Policy. 2008;13(2):92-8.

63. Tong A, Sainsbury P, Craig J. Consolidated criteria for reporting qualitative research (COREQ): a 32-item checklist for interviews and focus groups. Int J Oual Health Care. 2007:19(6):349-57.

64. Gouvernement du Québec. Les services intégrés en périnatalité et pour la petite enfance à l'intention des familles vivant en contexte de vulnérabilité - cadre de référence. Québec: Ministère de la Santé et des Services sociaux; 2004.

65. Ministère de la Santé et des Services sociaux. Naître égaux - Grandir en santé. Un programme intégré de promotion de la santé et de prévention en périnatalité. Québec: Ministère de la Santé et des Services sociaux; 2002.

66. Ministère de la Santé et des Services sociaux. Programme national de santé publique 2015-2025. Québec: Gouvernement du Québec; 2015.

67. Elo S, Kyngäs H. The qualitative content analysis process. J Adv Nurs. 2008; 62(1):107-15.

68. Borgatti SP, Everett MG, Freeman LC. Ucinet for windows: software for social network analysis. Harvard: Analytic Technologies; 2002

69. Scott J. Social network analysis. 3rd ed. Thousand Oaks: Sage; 2013.

70. Poh HL, Koh SS, He HG. An integrative review of fathers' experiences during pregnancy and childbirth. Int Nurs Rev. 2014;61(4):543-54 